\title{
The verbal-loop hypothesis (VLH): A within-subject study with a perceptual recognition task
}

\section{L. SANG and JOHN ROSS, University of Western Australia, Nedlands, Western Australia 6009}

The verbal-loop hypothesis (VLH) was examined, using a within-S design, a perceptual recognition task, and a set of patterns of respectable pictorial quality. No significant relationship between verbalization and recognition accuracy was found.

Glanzer \& Clark $(1962,1963,1964)$ have published three sets of studies, all of which are taken to provide evidence favoring their "Verbal-Loop Hypothesis" (VLH). The hypothesis states that Ss remember visual displays by translating them into words and using the verbal translation in carrying out subsequent memory tasks. Pattern complexity, under this hypothesis, is indexed by the length of the S's description: the longer the description, the more complex the figure, and therefore the greater the perceptual memory difficulty.

The evidence put forward by Glanzer \& Clark may be criticized on statistical grounds, since it presents correlations of average verbal length scores with average reproduction scores. Ross (1968) showed that Glanzer \& Clark's method provides an inflated estimate of the correlation necessary to test their hypothesis.

Glanzer \& Clark's conclusions may also be limited by their special kinds of stimuli (sequences of familiar or binary figures and runs of binary digits) and by their special kind of "reproduction" method, in which Ss are required to write down descriptions of pictures in recall.

This paper reports the results of studies that employ a within-S design to obtain a proper estimate of correlation between verbal-description length and perceptual-memory difficulty and that use recognition of pictorial designs to measure perceptual-memory difficulty.

Preliminary work showed that it seems possible to separate judged visual complexity from verbal-description lengths. For example, there seem to exist patterns that are difficult to verbalize but easy to visualize. In the present study, patterns that vary widely and somewhat orthogonally in visual complexity, as well as in difficulty of description, and a perceptual-recognition task are used.

\section{EXPERIMENT 1}

The aims of this experiment are: (1) to determine if there is a common basis for judgment of difference between patterns, and (2) to establish suitable distractors for a subsequent perceptual-recognition memory task.

\section{Subjects}

The Ss $(N=10)$ were senior students at the University of Western Australia whose ages ranged from 20 to 23 years.

\section{Materials}

Twenty-four sets of line figures and separate sets each of eight distractors for each figure were drawn with black India ink (except for colored distractors that were drawn in red) in solid lines on white cardboard sheets, $7 \times 5$ in. The figures were about $2 \mathrm{in}$. in width and $1 \mathrm{in}$. in height and were centered. There were 216 figures in all, counting the original figures and the distractors.

The distractors were generated from the originals by rotation, by reflection, or by changing some details of the original. Each distractor was intended to differ from its original in a specified manner.

\section{Procedure}

From each of the 24 sets of originals and distractors, a standard pair was chosen, consisting of the original figure and one distractor of that particular set. The different standard pairs for the different sets were picked by the $E$, and each was used to represent a difference of 10 units.

The Ss were asked to perform two tasks.

In Task 1, Ss made magnitude estimates of differences between the original figure and each of the distractors, respectively. Each judgment was based on a value of 10 for the difference between the members of the standard pair.
In Task 2, the same Ss were asked to make magnitude estimates of difference for the 24 different standard pairs, with one of the standard pairs being used as standard and assigned a value of 10 . These judgments were later used to adjust scale-unit differences in the Ss' judgments in Task 1, due to differences between the standard pairs in terms of which separate stimulus sets were judged.

\section{Results and Discussion}

A matrix of correlations between Ss was obtained for each set of figures and factor analyzed.

On the average, for each set of figures, the first factor accounted for about $49 \%$ of the variance, the second another $23 \%$, and the next eight factors for a further $28 \%$. Judging from the size of the latent roots, it seems that there are only two factors of any significance. Factor plots were drawn showing the position of Ss in the solution for each set of figures. It is striking that for 21 of 24 figures, the configuration of the Ss on the first factor is remarkably similar. It is less so on the second factor.

A reasonable interpretation seems to be that the first factor represents a common basis for the estimation of differences used by most Ss, and that the second factor represents an idiosyncratic or minority basis used by some Ss for particular pattern sets. It is worth noting that it is not always the same Ss who fall away from the main cluster on Factor 1.

To find suitable original-distractor pairs, a pool was formed, separately for each set of figures, of Ss who appeared from the factor analytic results to be using a common basis for the estimation of differences. Pairs of figures were chosen for which the pool's mean adjusted magnitude estimate of differences was near 10 , and for which the standard deviation was small.

For 9 of the 24 sets, no suitable original-distractor pair could be found. The means and standard deviation for the 15 sets for which suitable original-distractor pairs were available are given in Table 1 , and the pattern pairs themselves are shown in Fig. 1 .

\section{EXPERIMENT 2}

The aim of this experiment was to examine the VLH directly by using a within-S design and a recognition task

Table 1

Mean Adjusted Magnitude Estimates of Difference, Standard Deviations, and Correlations Between Accuracy of Recognition and Verbalization for Original-Distractor Pairs

\begin{tabular}{|c|c|c|c|c|c|c|c|c|c|c|c|c|c|c|c|}
\hline $\begin{array}{l}\text { Original- } \\
\text { Distractor } \\
\text { Pair }\end{array}$ & 1 & 2 & 3 & 4 & 5 & 6 & 7 & 8 & 9 & 10 & 11 & 12 & 13 & 14 & 15 \\
\hline Mean & 11.09 & 7.96 & 13.11 & 11.72 & 12.01 & 6.27 & 8.79 & 10.53 & 7.82 & 10.37 & 10.11 & 12.01 & 10.26 & 12.25 & 9.50 \\
\hline SD & 7.41 & 4.71 & 1.94 & 2.88 & 2.24 & 4.62 & 3.51 & 4.15 & 2.20 & 5.44 & 5.48 & 4.81 & 5.79 & $3.7:$ & 4.27 \\
\hline Correlation & .06 & -.19 & -.12 & -.11 & .16 & -.08 & -.17 & .15 & .13 & -.06 & -.15 & -.29 & -.08 & .10 & .06 \\
\hline
\end{tabular}




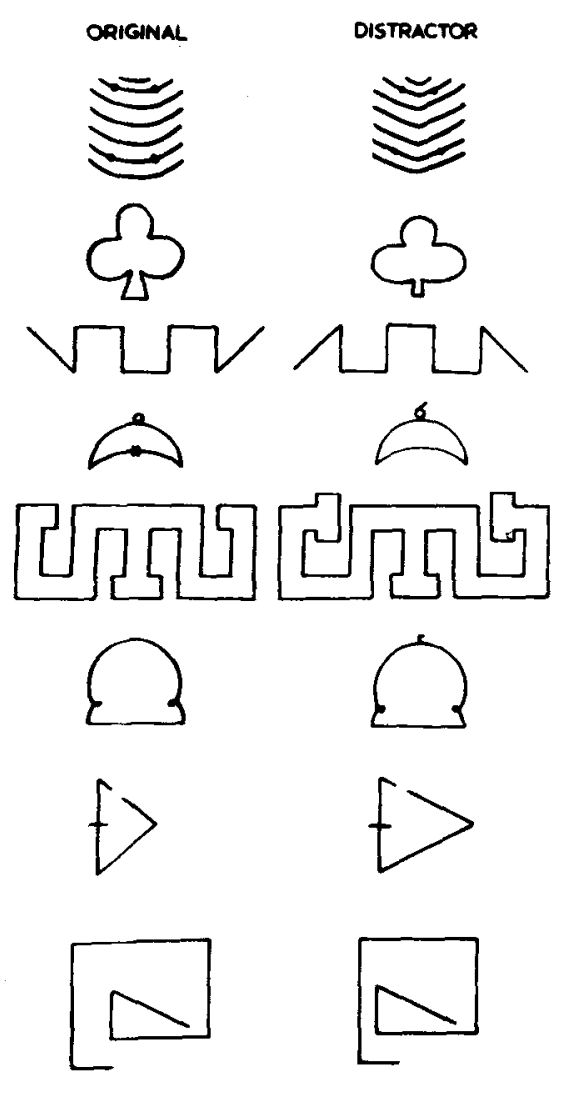

using pairs of original and distractor patterns obtained from Experiment 1.

Scores on the recognition task served as indices of perceptual memory for a pattern and were correlated with the lengths of verbalizations of the patterns. The VLH would predict a high negative correlation since, according to the hypothesis, the more words a $S$ uses to verbalize the pattern, the more complex the pattern is for him and the less accurate his performance.

\section{Subjects}

The $\mathrm{Ss}(\mathrm{N}=27)$ were volunteer first-year psychology students at the University of Western Australia. Their ages ranged from 17 to 22 years.

\section{Material}

Fifteen original patterns and their 15 distractors (Fig. 1) were photographed on $35-\mathrm{mm}$ slides. A $35-\mathrm{mm}$ Leizt projector projected the patterns onto a $4 \times 5 \mathrm{ft}$ screen in a semi-darkened room. An Alphax shutter was fitted in front of the projector lens to control the exposure time of the pattern in the recognition task. Procedure

The Ss performed two tasks:

Recognition task. Ss were shown pairs of patterns, each consisting of an original followed either by itself or its distractor. Each pair occurred 10 times in a random

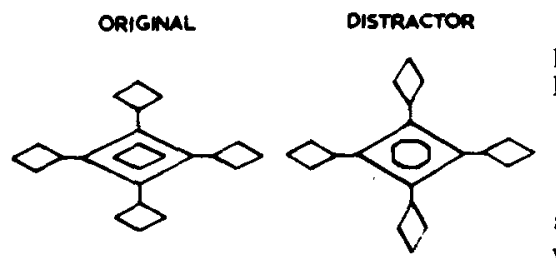

Fig. 1. Original pattern and distractor pairs, Nos. 1-8 in the left-hand column and Nos. 9.15 in the right-hand column.

Results

A correlation was calculated between accuracy of recognition and verbal-description length for each $S$. Accuracy of recognition was measured by number of correct judgments in each recognition task, and verbal-description length by number of words used in the verbalization task. Correlations were averaged over Ss using Fisher's $z$ transformation. The average correlation value was -.120 .

Mean accuracy scores and verbal-description lengths for each pattern were used to obtain a correlation between accuracy and verbal-description length that used averages over Ss. Predictably (Ross, 1968), the value, -.435 , is much higher than the average of the within-S correlations.

For each original-distractor pair, a correlation was calculated between accuracy of recognition and verbal-description length. The correlation values are reported in Table 1. Each is low in absolute value and some are positjve.

\section{GENERAL DISCUSSION}

Experiment 1 provided a set of 15 original-distractor pairs judged to differ in appearance by much the same amount. Experiment 2 used the pairs in a perceptual recognition task, remembering a picture and recognizing it again, the assumption being that within-S variation in task performance would reflect difficulty in remembering, since difficulty in discrimination between original and its distractor was controlled.

Experiment 2 showed that the within-S correlation between accuracy of recognition and verbal description length was low, the mean value being -.120 . The implication is that there is little connection for a given $S$ between the difficulty he has in remembering a pattern and the number of words he requires to describe it, contrary to the VLH. There is also little correlation when the variation is over Ss rather than over patterns (see Table 1). A person who requires a lot of words to describe a pattern is not a person who has more difficulty in remembering it. The last statement is also probably contrary to the VLH, but ambiguities in the hypothesis prevent any firm judgment on the point.

The only correlation that might indicate support for the VLH in the results presented is that between-S averages for accuracy and verbal description length, but, as Ross (1968) has pointed out, correlations based on averages cannot be lightly interpreted, since they almost 
always grossly overestimate the correlation about which the VIH makes a prediction.

It might be argued that unreliability has reduced the correlations reported to the low values they show, but reliabilities would need to be very low indeed, near .25 at most, to raise the values reported even as high as .5 , let alone to the values of .8 and .9 , on which Glanzer and Clark (1962, 1963 , 1964) base their conclusions. A supplementary study showed that the correlation between written and spoken description lengths, the two being obtained one week apart, is 59 , which suggests that reliability for written descriptions is reasonably high.

The results reported here show that there is little or no support for the VLH when statistical artifacts are removed from the picture and pictorial patterns are put in.

\section{REFERENCES}

GLANZER, M., \& CLARK, W. H. Accuracy of perceptual recall: An analysis of organization. Journal of Verbal Learning \& Verbal Behavior, 1962, 1, 289-299.

GLANZER, M., \& CLARK, W. H. The verbal-loop hypothesis: Binary numbers. Journal of Verbal Learning \& Verbal Behavior, $1963,2,301-309$.

GLANZER, M., \& CLARK, W. H. The verbal-loop hypothesis: Conventional figures. American Journal of Psychology, 1964, 77, 621-626.

ROSS, J. The verbal-loop hypothesis: A statistical audit. Australian Journal of Psychology, 1968, 20, 233-235.

\section{An explanation of the effect of tilt on the Poggendorff illusion}

\section{A. W. PRESSEY, University of Manitoba, Winnipeg 19, Canada}

An expalanation of the interaction between the effect of tilt and orientation on the Poggendorff illusion is given in terms of three facts: (1) that the illusion decreases with a decrease in distance between the parallel lines, (2) that the illusion increases as the angle of the oblique decreases, and (3) that the retinal image changes systematically as the target is tilted backwards.

In 1966, Leibowitz and Toffey measured the Poggendorff illusion as a joint function of orientation and tilt of the target. Although tilt was found to interact with orientation, they did not offer any explanation of their results. The present report is an attempt to account for Leibowitz and Toffey's findings on the basis of three well known facts. The first is that the Poggendorff illusion increases as the angle that the interrupted oblique forms with the parallel line decreases. The second fact is that the Poggendorff illusion decreases as the distance between the parallel lines decreases. And the third is that systematic changes in the size of the retinal image occur as a target is tilted backwards. The general rule is that vertical distances decrease, but horizontal distances remain constant.

The four orientations of the Poggendorff illusion used by Leibowitz and Toffey are shown in Fig. 1 ( $A$ to $D$ ). In addition, $3 \mathrm{deg}$ of tilt $(0,55$, and 80$)$ were employed in a factorial design. The results showed that tilt had no effect on either Fig. $1 \mathrm{~A}$ or $1 \mathrm{D}$, but the illusion decreased with increase of tilt in Fig. 1C. On the other hand, the Poggendorff illusion increased as Fig. 1B was tilted backwards.

Now consider the changes that occur in the retinal image as Fig. 1 $\mathrm{A}$ is tilted. The distance between the parallel lines remains the same, and the distance defining the angles of the oblique (e in Fig. 1A) also remains constant because both of these are horizontal distances. Therefore, the illusion should not change as the target is tilted. In Fig. 1D, tilting the target backwards results in a decrease of the distance between the two parallel lines that produces a decrease in illusion. However, the distance $\mathrm{h}$, which defines the angle of the oblique, decreases (the angle becomes more acute), and this results in an increase in illusion. Therefore, as the target is tilted, there are two contradictory trends, which probably cancel each other out to produce no effect.

Tilting Fig. $1 B$ results in the distance $f$ being reduced, which produces a more acute angle. The distance $j$, however, remains the same. Therefore, the illusion increases with an increase in tilt. Finally, tilting Fig. 1C decreases the distance k, but the angle remains constant. Hence, the illusion decreases with an increase in tilt. It will be noted that all the predictions derived from the changes in the retinal image that are consequent upon tilt are substantiated by the results reported by Leibowitz and Toffey.

\section{REFERENCE}

LEIBOWITZ, H., \& TOFFEY, S. The effect of rotation and tilt on the magnitude of the Poggendorff illusion. Vision Research, 1966, 6, 101-103.
A

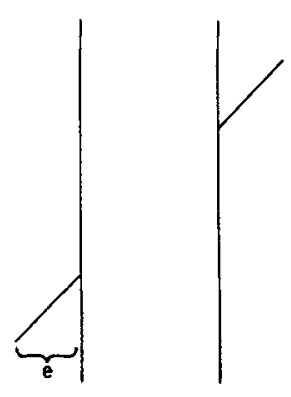

B

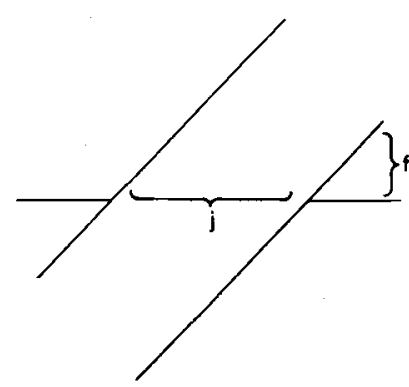

C

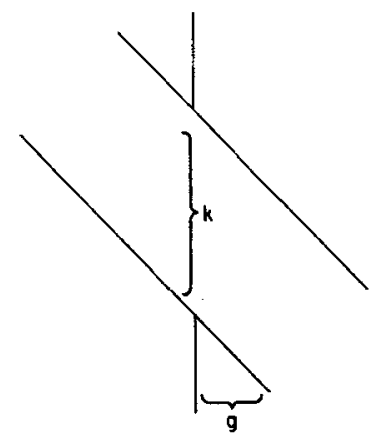

D

Fig. 1. Orientations of the Poggendorff illusion employed by Leibowitz \& Toffey (1966). 